\title{
From microdroplets to microparticles: organic particles crystallization by Spray Flash Evaporation
}

\author{
Yan Busby*, Fabien Schnell, Denis Spitzer \\ ${ }^{1}$ Nanomatériaux pour les Systèmes Sous Sollicitations Extrêmes (NS3E), UMR 3208, \\ French-German Research Institute of Saint-Louis, 68301 Saint-Louis, France \\ *Corresponding author email: busbyan@gmail.com
}

\begin{abstract}
Caffeine crystals are formulated through an aerosol-based vacuum process called spray flash evaporation (SFE). The spray conditions such as the injected solution temperature (70-150 ${ }^{\circ} \mathrm{C}$ ) and pressure (20-40 bar) can be tuned to ensure being in flash evaporation conditions in order to (i) reduce the droplets size down to the micron range and (ii) to speed up the solvent evaporation to promote the in-flight crystallization of micro and submicron particles. This manuscript present phase Doppler particle analysis (PDPA) results showing the droplets size, velocity and evaporation rate of pure-solvent aerosols. These data are used to correlate the process parameters to the final particles size and morphology as shown by electron microscopy images. For $1 \%$ caffeine solutions in ethanol and dichloromethane, the solvent evaporation rate, viscosity and the solution temperature are found to reduce the droplet size and so the formed caffeine rods length, however, the particles continue to grow in the filter because of non-fully evaporated droplets impacting on pre-formed primary crystals. Multiple metrologies are combined to investigate the particles morphology and structure.
\end{abstract}

\section{Keywords}

spray flash evaporation, PDPA, nanoparticles, organic crystals

\section{Introduction}

Many efforts are directed to improve methods for the control the nucleation and growth of crystalline organic nanoparticles. In particular, aerosol-based methods show multiple advantages such as low solvent consumption, few processing steps, easy particle collection by filtering and high production rate thanks to the possibility of operating in continuous-flow conditions. It is highly desirable to further develop aerosol-based technologies for the crystallization of controlled organic submicron structures.[1-3]

In this work we explore the formulation of organic crystals through an easy and continuous flow processing technology that combines high rate, low energy consumption and a minimum environmental impact called spray flash evaporation (SFE). This technology has been already demonstrated for the crystallization of submicron energetic materials and pharmaceutics.[4-6] The SFE process consists in injecting a pressurized and superheated solution into a primarypumped vacuum reactor (mbar) to promote the rapid solvent evaporation leading to the crystallization of the solute into small organic particles. The powder material is collected by a mechanical filter (Figure 1a).

The flash evaporation process occurs because at the injection temperature (pressure) the reactor pressure is lower (higher) than the liquid saturation pressure; in these conditions, the pressure drop (tension, $\Delta \mathrm{P}$ ) and the superheat $(\Delta \mathrm{T})$ promote the growth and burst of bubbles inside the liquid phase at the injection nozzle which results in the formation of a fine aerosol in which micro droplets undergo an ultrafast evaporation (Figure 1b and 1c).[7-9] 
In order to further develop the SFE technology and to be able to control the formulation of more complex binary particles [10-12] in particular by spraying solutions containing multiple organic precursors, we have performed a systematic experimental study on model solvents and a model solution in order to correlate the main SFE process parameters to the aerosol droplets and the particles and aggregates properties.

(a)

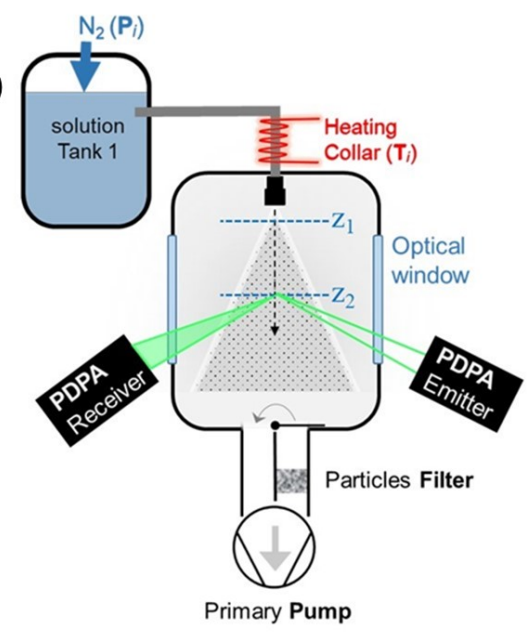

(b)

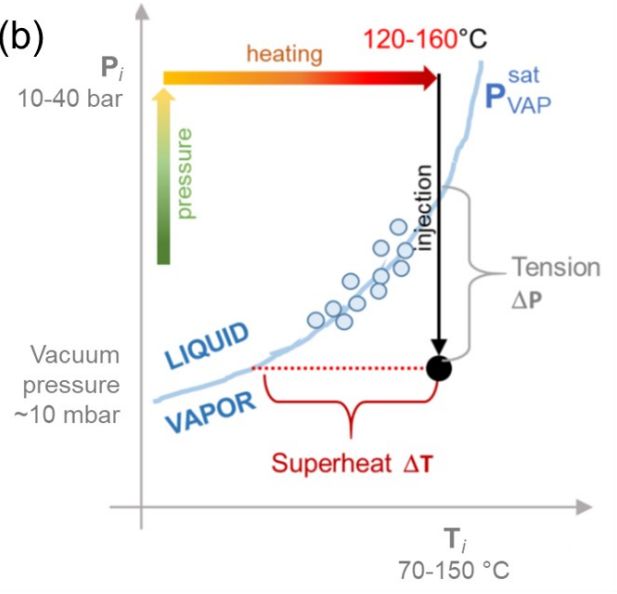

(c)

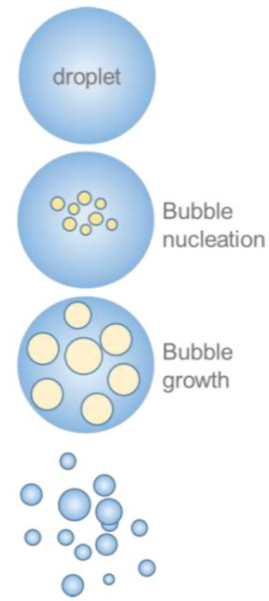

Figure 1. (a) spray flash evaporation setup: a superheated $\left(T_{i}\right)$ and pressurized $\left(P_{i}\right)$ liquid solution is injected through a nozzle into a primary pumped reactor. The droplet size and velocity are measured by PDPA in a $120^{\circ}$ geometry and crystals are collected by switching the aerosol flux through a mechanical filter. (b) definition of flash

evaporation superheat and tension. (c) Pictorial scheme of bubble nucleation and growth in the nozzle.

Specifically, our approach is to analyse solvent aerosols to correlate the solvent properties with the droplets size, velocity as a function of the injected liquid pressure $\left(\mathrm{P}_{\mathrm{i}}\right)$ and temperature $\left(T_{i}\right)$. Furthermore, the solvent evaporation rate is also estimated from the average droplet size reduction along its axial path. These data are used to interpret the crystallization mechanism of model particles during the SFE process.

The particles growth is investigated by spraying a diluted caffeine $\left(\mathrm{C}_{8} \mathrm{H}_{10} \mathrm{~N}_{4} \mathrm{O}_{2}\right)$ solution; caffeine was chosen for its typical rod-like (1D) growth mechanism.[13-14] We selected two solvents, dichloromethane (DCM) and ethanol, which have been chosen for their wellseparated physicochemical properties and caffeine solubility values (about $140 \mathrm{mg} / \mathrm{mL}$ in $\mathrm{DCM}$ and $15 \mathrm{mg} / \mathrm{mL}$ in ethanol). Morphological characterizations of caffeine crystals is performed by electron microscopy, Raman spectroscopy and X-ray diffraction (XRD) which have contributed to shine light on the nucleation and growth mechanisms during the SFE process.

\section{Material and Methods}

The SFE setup (Figure 1) consist of a nitrogen-pressurized reservoir tank connected to the main vacuum reactor chamber connected to a high-capacity primary pump $\left(95 \mathrm{~m}^{3} / \mathrm{h}\right)$. A heating collar, driven by a PID controller, regulates the temperature of the liquid just before entering the injection nozzle orifice (BETE). The nozzle ensures the generation of a full-cone foggy aerosol with micron sized droplets characterized by a reduced liquid flow rate $(0.5-1$ $\mathrm{L} / \mathrm{h}$, depending on $\mathrm{P}_{\mathrm{i}}$ ). During the spraying, the reactor pressure increases from the base pressure of $0.1 \mathrm{mbar}$ to $\sim 10-20$ mbar depending on $\mathrm{Pi}$. 
The main chamber is equipped with $100 \mathrm{~mm} \mathrm{~N}-B K 7$ windows with $120^{\circ}$ angle alignment which are used to perform in-situ PDPA measurements (Dual PDA FlowExplorer, Dantec Dynamics). The droplet size distribution is measured at 1 and $2 \mathrm{~cm}$ axial distance from the nozzle to estimate the evaporation rate.

Caffeine crystals are formed by spraying Ethanol/Caffeine and DCM/Caffeine solutions at constant pressure and variable temperature ( $T_{i}$ from 120 to $\left.140^{\circ} \mathrm{C}\right)$. Products were collected by switching the aerosol flux through a home-designed mechanical filter with micron sized mesh during a filtering time variable from 1 to $20 \mathrm{~min}$. The collection time was set to ensure a balance between achieving of a suitable product mass while limiting the solvent vapours exposure of the formed crystals. The particle's morphology is investigated by field emission gun scanning electron microscopy on gold metallized samples (SEM, FEI Nova NanoSEM 450, Hillsboro, Oregon, USA) operated at $10 \mathrm{kV}$. To prevent surface charging, caffeine particles were sputtered with $10 \mathrm{~nm}$ (nominal thickness) gold prior to the analysis. The structure and polymorphs are investigated by Raman spectroscopy (LabRam HR evolution, Horiba, Japan) and X-ray diffraction (AXS Advance D8, Bruker, Germany).

\section{Results and Discussion}

(a) Droplet size distribution in pure-solvent aerosols

The droplet size distribution of ethanol and DCM aerosols at $2 \mathrm{~cm}$ from the injection nozzle is derived from in-situ PDPA, for a range of $70<\mathrm{T}_{\mathrm{i}}<140^{\circ} \mathrm{C}$ and $10<\mathrm{P}_{\mathrm{i}}<40$ bar values. The droplet size is mainly affected by the superheat value with a pronounced increase of the number of micron sized droplets. The injection pressure also favours the formation of smaller droplets (Figure 2a). Unfortunately, the distribution of submicron cannot be directly counted by PDPA, however, information can be extrapolated by the lineshape of the distribution curves.

Dichloromethane features a more compact distribution with $30 \%$ smaller average droplet size. This is explained by the higher volatility of DCM as compared to ethanol, which improves the flash evaporation process. As compared to ethanol, DCM has $(i)$ a lower viscosity $(\sim 0.3 / 1.2$ $\mathrm{mPa} . \mathrm{s}),($ ii) a higher vapour pressure ( $48 / 6 \mathrm{KPa})$ and (iii) a lower boiling temperature (40 / $80^{\circ} \mathrm{C}$ ). The physical-chemical properties of DCM allow thus for a higher bubbling of the solvent when it reaches the nozzle, a more effective atomization and a faster solvent evaporation.

The droplet evaporation rate is estimated from the reduction of the average droplets size when travelling along the axial path. From the analysis of data at $\mathrm{T}_{\mathrm{i}}=140{ }^{\circ} \mathrm{C}$ and $\mathrm{P}_{\mathrm{i}}=20$ bar, we observe an average droplet size reduction, after travelling for $1 \mathrm{~cm}$ between 7 and $8 \%$, with an average diameter reduction from 1.8 to $1.65 \mu \mathrm{m}$ for DCM (Figure $\mathbf{2 b}$ ). The droplet velocity mainly depends from the pumping capacity and varies from 50 to $200 \mathrm{~m} / \mathrm{s}$ with an average value at $160 \mathrm{~m} / \mathrm{s}$. From these data, it is possible to estimate the DCM droplet evaporation rate at $1.10^{-10} \mathrm{~L} / \mathrm{s}$ (and slightly lower for ethanol). This value implies that a "slow" $(50 \mathrm{~m} / \mathrm{s}$ ) spherical droplet of $1 \mu \mathrm{m}$ diameter will evaporate in $\sim 40 \mu$ s after having travelled approximately $0.2 \mathrm{~cm}$ inside the SFE reactor. However, a fast $(200 \mathrm{~m} / \mathrm{s})$ and larger droplet of $5 \mu \mathrm{m}$ diameter will completely evaporate in $\sim 6 \mathrm{~ms}$ after having travelled a distance of $1.2 \mathrm{~m}$. In our setup, the filter is placed at about $1 \mathrm{~m}$ distance from the injection nozzle, so it is reasonable to expect that some droplets will not fully evaporate before reaching the filter. If droplets reach the filter, its surface would be cooled by the solvent evaporation, thus favouring the condensation of solvent vapours. 

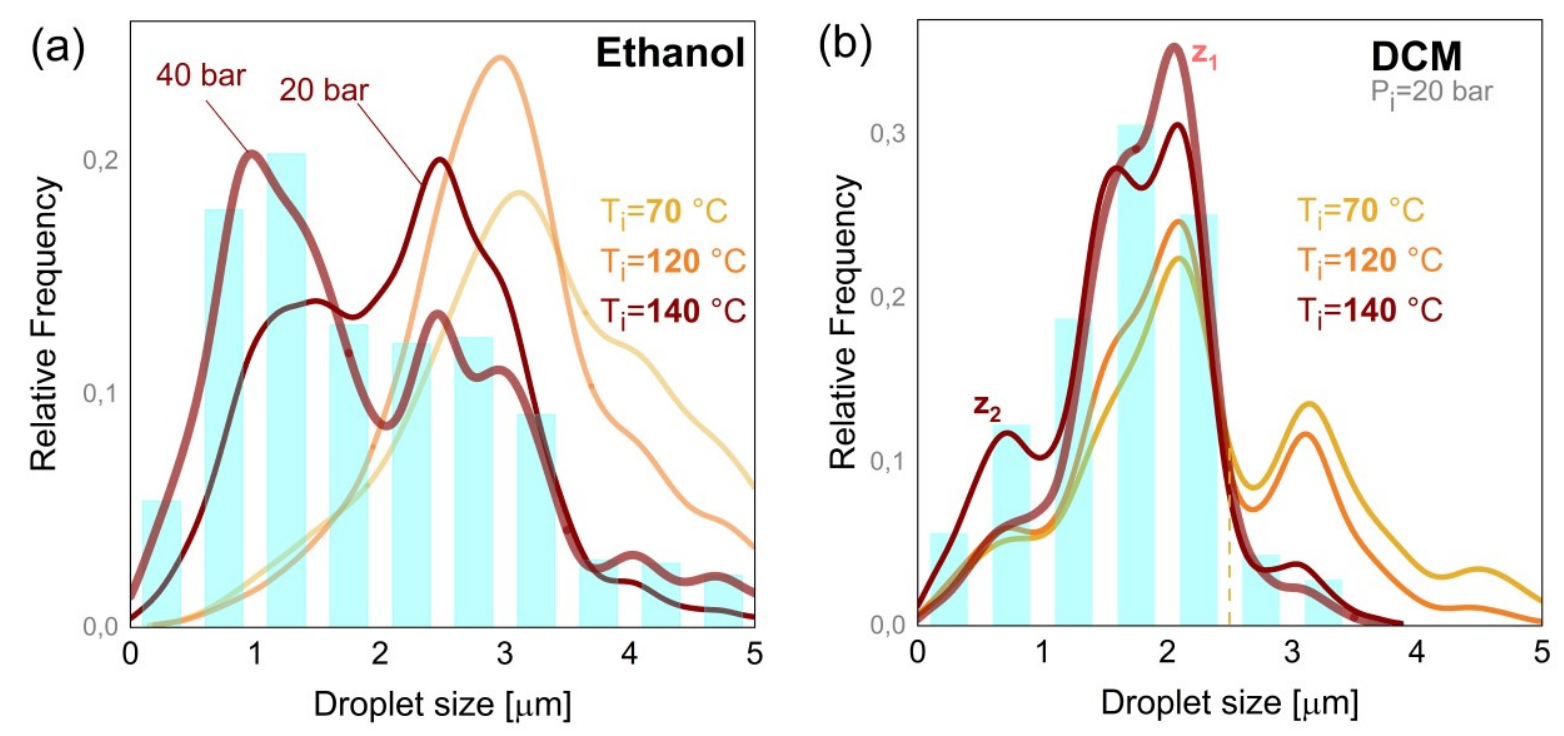

Figure 2. Droplet size distribution for ethanol (a) and dichloromethane (b) solvents for various $P_{i}$ and $T_{i}$ spray flash evaporation parameters derived from in-situ PDPA.

(b) Caffeine particles characterization

The correlation between the SFE process parameters and the morphology of caffeine crystals, is shown in Figure 3.

For a 1 wt. \% solution in ethanol, caffeine crystals collected after 1 min (30 bar, $140^{\circ} \mathrm{C}$ ) feature the typical rod-like morphology with a diameter of $200 \mathrm{~nm}$ and length between 1 and $5 \mu \mathrm{m}$ (Figure 3a).

Interestingly, the rod length increases to $20-30 \mu \mathrm{m}$ when the collection is extended to $10 \mathrm{~min}$ (Figure 3b), suggesting that part of the crystal growth and agglomeration occurs in the filter. If the caffeine concentration is lowered to $0.3 \mathrm{wt} \%$ (Figure $3 \mathrm{c}$ ), the production rate slows down and the nucleation of primary crystals inside the droplet microreactor is retarded. As a consequence, a longer collection time $(20 \mathrm{~min})$ is needed to obtain a similar amount of powder in the filter and a comparable length respect to particles in Figure $3 \mathrm{~b}$ but with a larger agglomeration of particles.

For caffeine crystals formulated in DCM (Figure 3d) the flash conditions ensure having a large amount of $1 \mu \mathrm{m}$ or submicron droplets which fully evaporate before reaching the filter. While the average rod length is similar to the one obtained with ethanol, we observe the presence of submicron caffeine crystals decorating larger rods; this feature was not observed in crystals formulated ethanol.

Our interpretation is that these correspond to primary crystals formed from small droplets which fully evaporate before reaching the filter. Successively, the particles grow in the filter due to the impact of (larger) solution droplets.

Confocal Raman and XRD analyses show that for that samples are anhydrous caffeine crystals in $\beta$-phase as the precursor powder. The possibility of tuning the polymorph will be will be explored in the future for more appropriate organic crystals. 
(a)

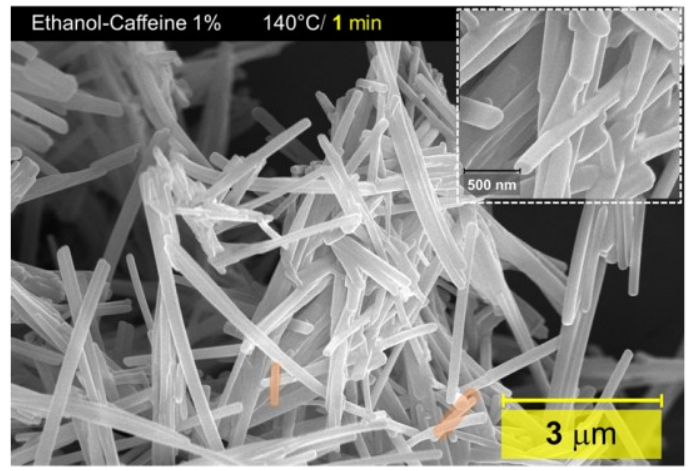

(c)

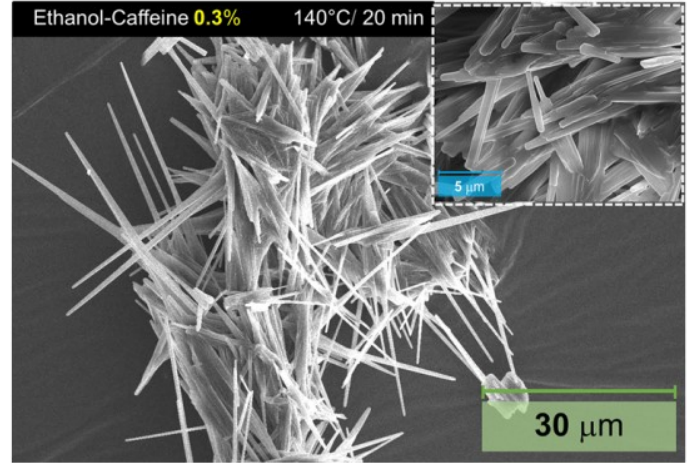

(b)

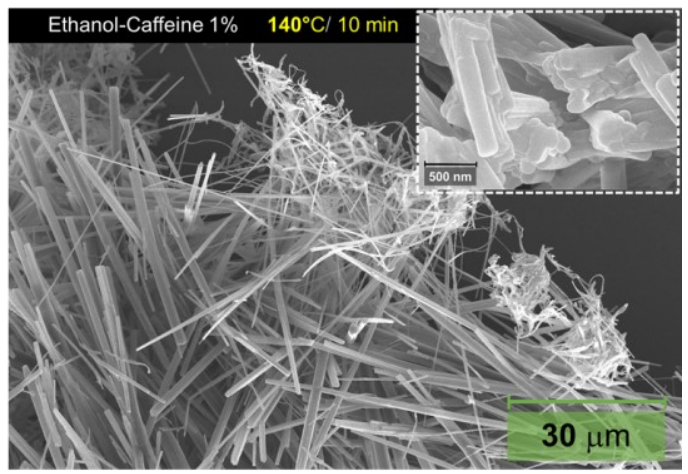

(d)

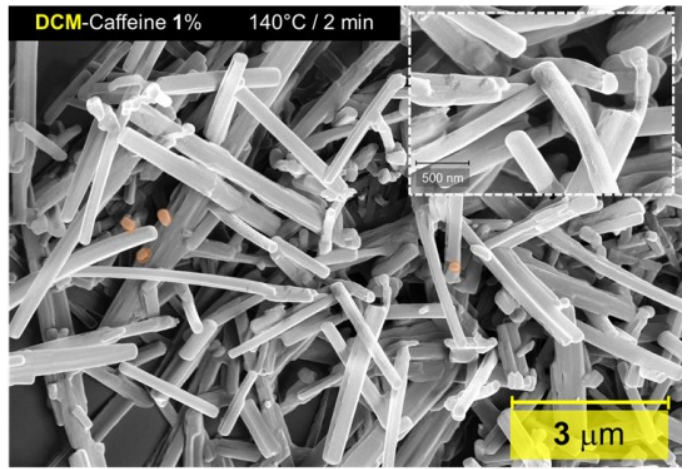

Figure 3. Morphology if caffeine crystals formulated by SFE in ethanol (a-c) and dichloromethane (d). The concentration $(0.3,1 \%)$ the injection temperature and the collection time are indicated in each panel.

\section{Conclusions}

the growth mechanism of caffeine crystals by SFE, an aerosol-based technology based on vacuum flash evaporation are investigated by in-situ phase Doppler particle analysis on two solvent droplets as a function of the flash process parameters ( $\mathrm{Pi}, \mathrm{Ti})$. We evidence the strong role of (i) the injection temperature in lowering the droplet size, (ii) the pumping capacity on the droplet velocity and we measured the evaporation rate which allows to predict if droplets are fully evaporated before reaching the filter. We obtain anhydrous caffeine crystals in $\beta$ phase with rod lengths between 1 and $30 \mu \mathrm{m}$. Results point out that the nucleation of primary particles occurs in flight the droplet microreactor while the rod growth and agglomeration mainly happen in the filter because of non-fully evaporated droplets.

We believe this systematic work will help for the better understanding control and widespread of aerosol-based crystallization technologies for the viable formulation of complex organic nanostructures

\section{Acknowledgments}

The authors thank the Direction Générale de l'Armement (DGA, France) and the Bundesamt für Ausrüstung, Informationstechnik und Nutzung der Bundeswehr (BAAINBw, Germany). The NS3E Lab acknowledge the support from ISL, the CNRS and the University of Strasbourg.

\section{References}

[1] de Souza Lima, R., Ré, M.-I., and Arlabosse, P., 2020, "Drying Droplet as a Template for Solid Formation: A Review," Powder Technol., 359, pp. 161-171.

[2] Boel, E., Koekoekx, R., Dedroog, S., Babkin, I., Vetrano, M. R., Clasen, C., and Van den Mooter, G., 2020, "Unraveling Particle Formation: From Single Droplet Drying to Spray Drying and Electrospraying," Pharmaceutics, 12(7), p. 625.

[3] Fang, F., Li, M., Zhang, J., and Lee, C., 2020, "Different Strategies for Organic Nanoparticle Preparation in Biomedicine," ACS Mater. Lett., 2(5), pp. 531-549. 
[4] Spitzer, D., Risse, B., Schnell, F., Pichot, V., Klaumünzer, M., and Schaefer, M. R., 2014, "Continuous Engineering of Nano-Cocrystals for Medical and Energetic Applications," Sci. Rep., 4, pp. 4-9.

[5] Risse, B., Spitzer, D., Hassler, D., Schnell, F., Comet, M., Pichot, V., and Muhr, H., 2012, "Continuous Formation of Submicron Energetic Particles by the Flash-Evaporation Technique," Chem. Eng. J., 203, pp. 158-165.

[6] Pessina, F., Schnell, F., and Spitzer, D., 2016, "Tunable Continuous Production of RDX from Microns to Nanoscale Using Polymeric Additives," Chem. Eng. J., 291, pp. 12-19.

[7] Chen, Q., M, K. J., Li, Y., and Chua, K. J., 2018, "Experimental and Mathematical Study of the Spray Flash Evaporation Phenomena," Appl. Therm. Eng., 130, pp. 598-610.

[8] Liu, L., Bi, Q. C., and Li, H. X., 2009, "Experimental Investigation on Flash Evaporation of Saltwater Droplets Released into Vacuum," Microgravity Sci. Technol., 21(SUPPL. 1), pp. 255-260.

[9] Cheng, W. L., Chen, H., Hu, L., and Zhang, W. W., 2015, "Effect of Droplet Flash Evaporation on Vacuum Flash Evaporation Cooling: Modeling," Int. J. Heat Mass Transf., 84, pp. 149-157.

[10] Spitzer, D., Risse, B., Schnell, F., Pichot, V., Klaumünzer, M., and Schaefer, M. R., 2014, "Continuous Engineering of Nano-Cocrystals for Medical and Energetic Applications," Sci. Rep., 4, pp. 4-9.

[11] Hübner, J., Deckert-Gaudig, T., Glorian, J., Deckert, V., and Spitzer, D., 2020, "Surface Characterization of Nanoscale Co-Crystals Enabled through Tip Enhanced Raman Spectroscopy," Nanoscale, 12(18), pp. 10306-10319.

[12] Urano, M., Kitahara, M., Kishi, K., Goto, E., Tagami, T., Fukami, T., and Ozeki, T., 2020, "Physical Characteristics of Cilostazol-Hydroxybenzoic Acid Cocrystals Prepared Using a Spray Drying Method," Crystals, 10(4), p. 313.

[13] Apshingekar, P. P., Aher, S., Kelly, A. L., Brown, E. C., and Paradkar, A., 2017, "Synthesis of Caffeine/Maleic Acid Co-Crystal by Ultrasound-Assisted Slurry Co-Crystallization," J. Pharm. Sci., 106(1), pp. 66-70.

[14] Park, S.-J., and Yeo, S.-D., 2008, "Recrystallization of Caffeine Using Gas Antisolvent Process," J. Supercrit. Fluids, 47(1), pp. 85-92. 